\title{
FINITE DIFFERENCE SOLUTIONS OF THE NONLINEAR SCHRÖDINGER EQUATION AND THEIR CONSERVATION OF PHYSICAL QUANTITIES *
}

\author{
CLEMENS HEITZINGER ${ }^{\dagger}$, CHRISTIAN RINGHOFER ${ }^{\ddagger}$, AND SIEGFRIED SELBERHERR ${ }^{\S}$
}

\begin{abstract}
The solutions of the nonlinear Schrödinger equation are of great importance for ab initio calculations. It can be shown that such solutions conserve a countable number of quantities, the simplest being the local norm square conservation law. Numerical solutions of high quality, especially for long time intervals, must necessarily obey these conservation laws. In this work we first give the conservation laws that can be calculated by means of Lie theory and then critically compare the quality of different finite difference methods that have been proposed in geometric integration with respect to conservation laws. We find that finite difference schemes derived by writing the Schrödinger equation as an (artificial) Hamiltonian system do not necessarily conserve important physical quantities better than other methods.
\end{abstract}

Key words. nonlinear Schrödinger equation, finite difference scheme, conservation law, variational symmetry

AMS subject classifications. 35Q55, 35L65, 65M06

\section{Introduction}

The wave equation, or the time-dependent Schrödinger equation,

$$
\mathrm{i} \hbar \frac{\partial \psi(\mathbf{r}, t)}{\partial t}=-\frac{\hbar^{2}}{2 m} \nabla^{2} \psi(\mathbf{r}, t)+V_{0} \cdot \psi(\mathbf{r}, t)
$$

describes the non-relativistic quantum mechanical properties for particles without spin. Here a particle of mass $m$ moves in a field represented by the potential energy function $V_{0}$. After scaling the $t$ and $\mathbf{r}$ coordinates, the one-dimensional Schrödinger equation takes the form

$$
\mathrm{i} u_{t}+u_{x x}+2 u V_{1}(t, x, u)=0
$$

where $V_{1}(t, x, u)$ denotes the potential. In the case of the Schrödinger equation with cubic nonlinearity in one space dimension, the potential is of the form $V_{1}(t, x, u)=$ $|u|^{2}+V(t, x)$ where $V(t, x)$ is an arbitrary real-valued function. Hence we consider numerical solutions of the initial-boundary value problem

$$
\begin{gathered}
u: \quad[0, T] \times[0,1] \rightarrow \mathbb{C}, \\
\Delta(t, x, u):=\mathrm{i} u_{t}+u_{x x}+2 \alpha u\left(|u|^{2}+V(t, x)\right)=0, \\
\alpha \neq 0, \\
u(0, x) \text { given, }
\end{gathered}
$$

periodic boundary conditions for $x \in[0,1]$.

*Received: March 7, 2007; accepted (in revised version): July 30, 2007. Communicated by Shi Jin. The first author acknowledges support by the Austrian Science Fund (Fonds zur Förderung der wissenschaftlichen Forschung, FWF) via an Erwin Schrödinger Fellowship."

${ }^{\dagger}$ Institute for Microelectronics, Technical University Vienna, A-1040 Vienna, Austria (Clemens. Heitzinger@TUWien.ac.at), corresponding author.

${ }^{\ddagger}$ Department of Mathematics, Arizona State University, Tempe AZ 85287, USA (Ringhofer@ ASU.edu).

§Institute for Microelectronics, Technical University Vienna, A-1040 Vienna, Austria (Siegfried. Selberherr@TUWien.ac.at). 
In Section 2 we calculate conservation laws by means of Lie Theory and Noether's Theorem [7-9]. After defining three finite difference schemes in Section 3, we discuss the numerical results for a recurrence solution in Section 4.

\section{Conservation laws}

To employ Lie theory and find the conservation laws we define $v:=\Re u$ and $w:=\Im u$ and have

$$
\begin{aligned}
& \Re \Delta=-w_{t}+v_{x x}+2 \alpha v\left(v^{2}+w^{2}+V(t, x)\right), \\
& \Im \Delta=v_{t}+w_{x x}+2 \alpha w\left(v^{2}+w^{2}+V(t, x)\right) .
\end{aligned}
$$

We can write the system as the first-order variational problem

$$
\mathcal{L}(u):=\int L\left(t, x, v, w, v^{(1)}, w^{(1)}\right) \mathrm{d} t \mathrm{~d} x
$$

with the Lagrangian

$$
L:=\frac{w v_{t}-v w_{t}}{2}-\frac{v_{x}^{2}+w_{x}^{2}}{2}+\alpha\left(\frac{v^{4}+w^{4}}{2}+v^{2} w^{2}+V\left(v^{2}+w^{2}\right)\right) .
$$

Hence we find for the Euler-Lagrange equations that $E_{v}(L)=\Re \Delta$ and $E_{w}(L)=\Im \Delta$ where $E_{v}$ and $E_{w}$ are Euler operators. A vector field

$$
\mathbf{v}=\tau(t, x, v, w) \partial_{t}+\xi(t, x, v, w) \partial_{x}+\varphi(t, x, v, w) \partial_{v}+\psi(t, x, v, w) \partial_{w}
$$

is a variational symmetry of $\mathcal{L}(u)=\int L \mathrm{~d} x$ iff [9]

$$
\operatorname{pr}_{n}(\mathbf{v})(L)+L \operatorname{Div}(\tau, \xi)=0 .
$$

Here $\operatorname{Div}(\tau, \xi)$ is the total divergence $\operatorname{Div}(\tau, \xi)=D_{t} \tau+D_{x} \xi$. In order to check this condition we use the relevantly prolonged operator

$$
\begin{aligned}
\operatorname{pr}_{1}(\mathbf{v})= & \tau(t, x, v, w) \partial_{t}+\xi(t, x, v, w) \partial_{x}+\varphi(t, x, v, w) \partial_{v}+\psi(t, x, v, w) \partial_{w} \\
& +\varphi^{t}(t, x, v, w) \partial_{v_{t}}+\psi^{t}(t, x, v, w) \partial_{w_{t}}+\varphi^{x}(t, x, v, w) \partial_{v_{x}}+\psi^{x}(t, x, v, w) \partial_{w_{x}},
\end{aligned}
$$

with

$$
\begin{aligned}
& \varphi^{t}(t, x, v, w)=D_{t}\left(\varphi-\tau v_{t}-\xi v_{x}\right)+\tau v_{t t}+\xi v_{t x}, \\
& \psi^{t}(t, x, v, w)=D_{t}\left(\psi-\tau w_{t}-\xi w_{x}\right)+\tau w_{t t}+\xi w_{t x}, \\
& \varphi^{x}(t, x, v, w)=D_{x}\left(\varphi-\tau v_{t}-\xi v_{x}\right)+\tau v_{t x}+\xi v_{x x}, \\
& \psi^{x}(t, x, v, w)=D_{x}\left(\psi-\tau w_{t}-\xi w_{x}\right)+\tau w_{t x}+\xi w_{x x}
\end{aligned}
$$

and solve (2.1) by equating the coefficients of the derivatives of the dependent variables to zero. We find that $\tau, \xi, \varphi$, and $\psi$ must satisfy the symmetry conditions

$$
\begin{aligned}
\tau(t) & =\tau(t) & & \text { arbitrary, } \\
\xi(x) & =\frac{1}{2} \tau_{t} x+a(t) & & a(t): \mathbb{R} \rightarrow \mathbb{R} \text { arbitrary } \\
\varphi(t, x, v, w) & =-\frac{1}{4} \tau_{t} v+\left(c-\frac{x^{2}}{8} \tau_{t t}-\frac{1}{2} a_{t} x\right) w & & c \in \mathbb{R} \text { arbitrary } \\
\psi(t, x, v, w) & =-\frac{1}{4} \tau_{t} w-\left(c-\frac{x^{2}}{8} \tau_{t t}-\frac{1}{2} a_{t} x\right) v, & &
\end{aligned}
$$




\begin{tabular}{llll} 
& Physical meaning & Var. symmetry & Conserved density \\
\hline$C_{1}$ & Charge, $V$ arbitrary & $\mathbf{v}_{1}=w \partial_{v}-v \partial_{w}$ & $P_{1}\left(\mathbf{v}_{1}\right)=\frac{1}{2}|u|^{2}$ \\
$C_{2}$ & Momentum, $V=V(t)$ & $\mathbf{v}_{2}=\partial_{x}$ & $P_{1}\left(\mathbf{v}_{2}\right)=\frac{1}{2}\left(v w_{x}-w v_{x}\right)=\frac{1}{2} \Im\left(u \bar{u}_{x}\right)$ \\
$C_{3}$ & Energy, $V=V(x)$ & $\mathbf{v}_{3}=\partial_{t}$ & $P_{1}\left(\mathbf{v}_{3}\right)=\frac{1}{2}\left(\alpha|u|^{2}\left(|u|^{2}+2 V\right)-\left|u_{x}\right|^{2}\right)$
\end{tabular}

TABLE 2.1. Variational symmetries and conserved densities. In all cases $\alpha \neq 0$ is arbitrary.

and

$$
\begin{aligned}
\frac{v \psi_{t}-w \varphi_{t}}{2 \alpha}=2 & \left(v^{2}+w^{2}+V\right)(v \varphi+w \psi)+\left(v^{2}+w^{2}\right)\left(\tau V_{t}+\xi V_{x}\right) \\
& +\left(\left(v^{4}+w^{4}\right) / 2+v^{2} w^{2}+V\left(v^{2}+w^{2}\right)\right)\left(\tau_{t}+\xi_{x}\right) .
\end{aligned}
$$

The outside potential $V(t, x)$ appears only in the last equation. We note that $\mathbf{v}=$ $w \partial_{v}-v \partial_{w}$ is a symmetry for arbitrary $\alpha$ and arbitrary outside potentials. Furthermore $\mathbf{v}=\partial_{t}$ is a symmetry provided that the outside potential is time-independent, i.e., $V_{t}=0$. Similarly $\mathbf{v}=\partial_{x}$ and $\mathbf{v}=t \partial_{x}-\frac{x w}{2} \partial_{v}+\frac{x v}{2} \partial_{w}$ are symmetries provided that the outside potential is space-independent, i.e., $V_{x}=0$. In both cases $\alpha \neq 0$ can be arbitrary.

Considering the case of a constant outside potential, $V \in \mathbb{R}$, we arrive at the symmetries

$$
\begin{aligned}
\tau(t) & =c_{3} & & c_{3} \in \mathbb{R} \text { arbitrary, } \\
\xi(x) & =c_{2}+c_{4} t & & c_{2}, c_{4} \in \mathbb{R} \text { arbitrary, } \\
\varphi(t, x, v, w) & =\left(c_{1}-\frac{1}{2} c_{4} x\right) w & & c_{1} \in \mathbb{R} \text { arbitrary, } \\
\psi(t, x, v, w) & =-\left(c_{1}-\frac{1}{2} c_{4} x\right) v & &
\end{aligned}
$$

that form a four-dimensional Lie algebra. After defining $\mathbf{v}_{i}$ to be the symmetry with $c_{i}=1$ and $c_{j}=0, j \in\{1,2,3,4\} \backslash\{i\}$, its commutator table is

$\begin{array}{lcccc} & \mathbf{v}_{1} & \mathbf{v}_{2} & \mathbf{v}_{3} & \mathbf{v}_{4} \\ \mathbf{v}_{1} & 0 & 0 & 0 & 0 \\ \mathbf{v}_{2} & 0 & 0 & 0 & -\frac{\mathbf{v}_{1}}{2} \\ \mathbf{v}_{3} & 0 & 0 & 0 & 0 \\ \mathbf{v}_{4} & 0 & \frac{\mathbf{v}_{1}}{2} & 0 & 0\end{array}$

We now use Noether's Theorem [8] to determine the conservation laws. In the case of a first-order variational problem the components of a conservation law $\operatorname{Div} P=0$ are in general given by [9]

$$
P_{i}(\mathbf{v})=\sum_{\alpha=1}^{q} \varphi_{\alpha} \frac{\partial L}{\partial u_{i}^{\alpha}}+\xi^{i} L-\sum_{\alpha=1}^{q} \sum_{j=1}^{p} \xi^{j} u_{j}^{\alpha} \frac{\partial L}{\partial u_{i}^{\alpha}} .
$$

In the case of an evolution equation with appropriate boundary conditions we find that the integral $\int_{\Omega} P_{1} \mathrm{~d} x$ of the conserved density $P_{1}$ is constant with respect to time on solutions of the PDE system.

The variational symmetries and their conserved density are summarized in Table 2.1. As for the physical interpretation, the rotation $\mathbf{v}_{1}=w \partial_{v}-v \partial_{w}$ corresponds to 
charge conservation, the translation $\mathbf{v}_{2}=\partial_{x}$ corresponds to momentum conservation, and the translation $\mathbf{v}_{3}=\partial_{t}$ corresponds to energy conservation, as usual in physical systems. It is clear from (2.2) that energy conservation arises from the symmetry $\mathbf{v}=\partial_{t}$ of an evolution equation in general.

\section{Three finite difference schemes}

We compare the following three finite difference schemes. The first scheme, $[\mathrm{H}]$, proceeds by writing the equation as a Hamiltonian system in canonical form in a straightforward manner [6]. The second method, $[\mathrm{P}]$, is based on the Ablowitz-Ladik model and a transformation to canonical form [1,4-6]. For the first two methods we use the 6 th order Gauß collocation method as the symplectic scheme. The third method, [RK], is a state-of-the-art Runge-Kutta method of order 6 .

3.1. Hamiltonian form. Depending on how the nonlinear term in (1) is discretized, one can write the system in Hamiltonian form in straightforward manner $\left(u \mapsto u_{k}\right)$ or one arrives at the Ablowitz-Ladik model $\left(2 u \mapsto u_{k-1}+u_{k+1}\right)$.

Discretizing the derivatives with respect to the space variable first in an equidistant manner, we obtain the equations

$$
\mathrm{i} \frac{\partial u_{k}}{\partial t}+\frac{u_{k+1}-2 u_{k}+u_{k-1}}{\Delta x^{2}}+2 \alpha u_{k}\left(\left|u_{k}\right|^{2}+V\left(t, x_{k}\right)\right)=0
$$

in the new variables $u_{k}, k \in\{1, \ldots, N\}$. After splitting $u_{k}$ into real and imaginary parts by $u_{k}=: v_{k}+\mathrm{i} w_{k}$ and introducing the notation $v:=\left(v_{1}, \ldots, v_{N}\right)$ and $w:=$ $\left(w_{1}, \ldots, w_{N}\right)$, we define the Hamiltonian

$$
H(v, w):=\frac{1}{\Delta x^{2}} \sum_{k=1}^{N}\left(v_{k} v_{k-1}-v_{k}^{2}+w_{k} w_{k-1}-w_{k}^{2}\right)+\frac{\alpha}{2} \sum_{k=1}^{N}\left(v_{k}^{2}+w_{k}^{2}+V\left(t, x_{k}\right)\right)^{2}
$$

and obtain the system $\left(\begin{array}{c}\dot{v} \\ \dot{w}\end{array}\right)=\left(\begin{array}{cc}0 & -I \\ I & 0\end{array}\right)\left(\begin{array}{c}\nabla_{v} H(v, w) \\ \nabla_{w} H(v, w)\end{array}\right)$ in canonical form.

3.2. Poisson form. We now discretize the nonlinear term using $2 u \mapsto u_{k-1}+$ $u_{k+1}$ and obtain

$$
\mathrm{i} \frac{\partial u_{k}}{\partial t}+\frac{u_{k+1}-2 u_{k}+u_{k-1}}{\Delta x^{2}}+\alpha\left(u_{k-1}+u_{k+1}\right)\left(\left|u_{k}\right|^{2}+V\left(t, x_{k}\right)\right)=0
$$

in the new variables $w_{k}, k \in\{1, \ldots, N\}$. Again we split $u_{k}$ into real and imaginary parts via $u_{k}=: v_{k}+\mathrm{i} w_{k}$ and introduce the notation $v:=\left(v_{1}, \ldots, v_{N}\right)$ and $w:=$ $\left(w_{1}, \ldots, w_{N}\right)$, which yields

$$
\left(\begin{array}{c}
\dot{v} \\
\dot{w}
\end{array}\right)=\left(\begin{array}{cc}
0 & -D \\
D & 0
\end{array}\right)\left(\begin{array}{c}
\nabla_{v} H(v, w) \\
\nabla_{w} H(v, w)
\end{array}\right)
$$

The entries of the diagonal matrix $D$ are $d_{k}:=1+\alpha \Delta x^{2}\left(v_{k}^{2}+w_{k}^{2}+V\left(t, x_{k}\right)\right)$ and

$H(v, w):=\frac{1}{\Delta x^{2}} \sum_{k=1}^{N}\left(v_{k} v_{k-1}+w_{k} w_{k-1}\right)-\frac{1}{\alpha \Delta x^{4}} \sum_{k=1}^{N} \ln \left(1+\alpha \Delta x^{2}\left(v_{k}^{2}+w_{k}^{2}+V\left(t, x_{k}\right)\right)\right)$.

This system is a Poisson system.

The transformation to canonical form is not unique and depends on $V$ (or generally on $V_{1}$ ), since $d_{k}$ depends on the potential. However the transformation to 
canonical form should be global, i.e., it should be identical for all time steps; otherwise poor performance for increasing time must be expected [5]. Hence we assume in the following that $V(t, x)=V(x)$ not depending on $t$.

In order to find a transformation to canonical form we employ the Darboux-Lie Theorem [6]. We set $\sigma(x):=\sqrt{\frac{\ln (1+x)}{x}}$ and $\tau(x):=\sqrt{\frac{\mathrm{e}^{x}-1}{x}}$. We use the transformation

$$
\begin{aligned}
p_{k} & :=v_{k} \sigma\left(\alpha \Delta x^{2}\left(v_{k}^{2}+w_{k}^{2}\right)\right), \\
q_{k} & :=w_{k} \sigma\left(\alpha \Delta x^{2}\left(v_{k}^{2}+w_{k}^{2}\right)\right)
\end{aligned}
$$

with its inverse

$$
\begin{aligned}
v_{k} & =p_{k} \tau\left(\alpha \Delta x^{2}\left(p_{k}^{2}+q_{k}^{2}\right)\right), \\
w_{k} & =q_{k} \tau\left(\alpha \Delta x^{2}\left(p_{k}^{2}+q_{k}^{2}\right)\right) .
\end{aligned}
$$

After this transformation the system is in canonical form and the new Hamiltonian $H$ in the variables $p$ and $q$ reads

$$
\begin{gathered}
H(p, q)=\frac{1}{\Delta x^{2}} \sum_{k=1}^{N} \tau\left(\alpha \Delta x^{2}\left(p_{k}^{2}+q_{k}^{2}\right)\right) \tau\left(\alpha \Delta x^{2}\left(p_{k-1}^{2}+q_{k-1}^{2}\right)\right)\left(p_{k} p_{k-1}+q_{k} q_{k-1}\right) \\
-\frac{1}{\alpha \Delta x^{4}} \sum_{k=1}^{N} \ln \left(\mathrm{e}^{\alpha \Delta x^{2}\left(p_{k}^{2}+q_{k}^{2}\right)}+\alpha \Delta x^{2} V\left(t, x_{k}\right)\right) .
\end{gathered}
$$

3.3. Runge-Kutta method of order 6 . The third method, [RK], is a stateof-the-art non-symplectic method that the symplectic schemes have to be compared with. We use the Hamiltonian form from Section 3.1 and a first-same-as-last embedded pair of explicit Runge-Kutta methods of order 6 including automatic time-step control. Although automatic time-step control is not easily achieved with symplectic integrators [5], it is an important feature of state-of-the-art (non-symplectic) methods.

\section{Numerical results}

In this section, we set $V:=0$ because of the restriction imposed by method $[\mathrm{P}]$. First we checked the correctness of the implementations of the three methods on several examples. The results for a square pulse initial condition are shown in Figures 4.1, 4.2, and 4.3. A time-step of $\Delta t:=1 / 50000$ was used on the interval $t \in[0,1 / 100]$, and a step-size of $\Delta x:=1 / 50$ was used on the interval $x \in[0,1]$. The differences between the three solution methods are shown in Fig. 4.4. Methods $[\mathrm{H}]$ and $[\mathrm{RK}]$ agree quite well (the difference is $4 \cdot 10^{-9}$ at most), whereas method $[\mathrm{P}]$ begins to deviate from the other two solutions (the point-wise difference is in the range of 0.003). As seen in the second example, the initial good agreement between methods $[\mathrm{H}]$ and $[\mathrm{RK}]$ is due to the small time-step used here.

Recently an optical Fermi-Pasta-Ulam recurrence [3] was demonstrated experimentally in an optical fiber $[2,10]$. For our numerical experiments we use the initial condition

$$
u(0, x):=\pi \sqrt{2}\left(1+\frac{1}{10} \cos (\pi x)\right)
$$

and periodic boundary conditions for $x \in[-1,1]$. In all calculations we use fixed-point iteration - as opposed to Newton iteration-because of its empirically found better convergence behavior for this kind of problem. The outside potential is assumed to 
784 FINITE DIFFERENCE SOLUTIONS OF THE NONLINEAR SCHRÖDINGER EQUATION

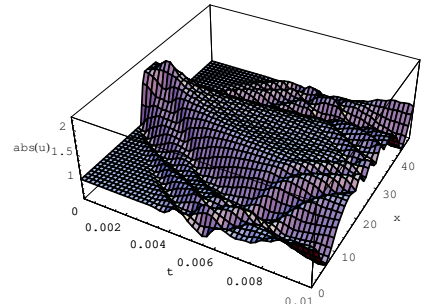

(a) Method $[\mathrm{H}]$.

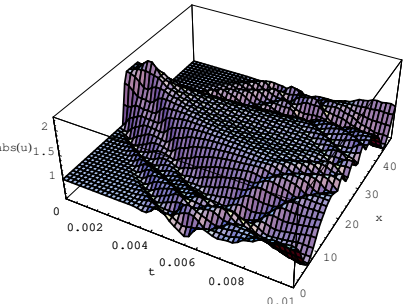

(b) Method [P].

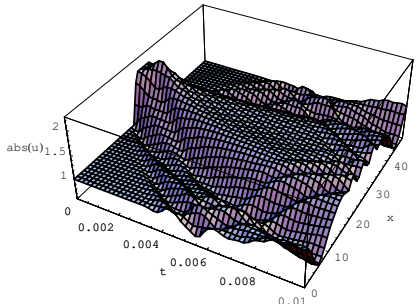

(c) Method $[\mathrm{RK}]$.

FIG. 4.1. The absolute value of the solution for a square pulse initial condition.

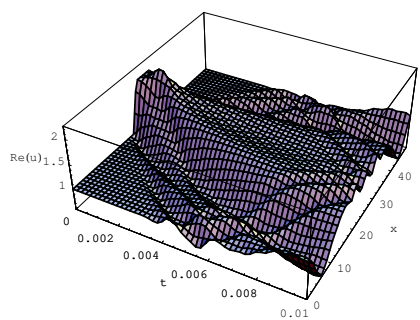

(a) Method $[\mathrm{H}]$.

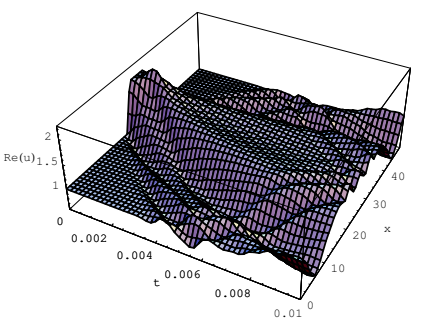

(b) Method [P].

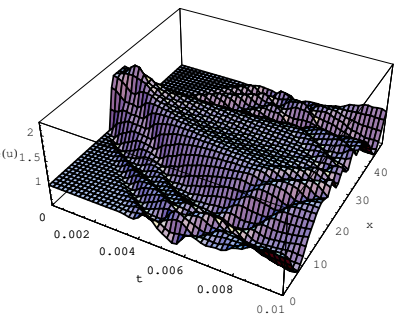

(c) Method $[\mathrm{RK}]$.

FIG. 4.2. The real part of the solution for a square pulse initial condition.

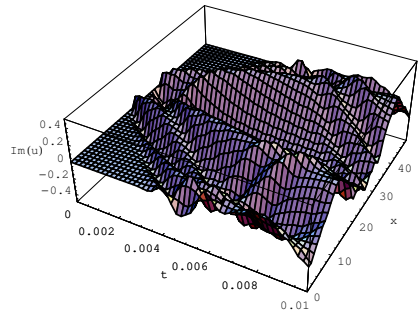

(a) Method $[\mathrm{H}]$.

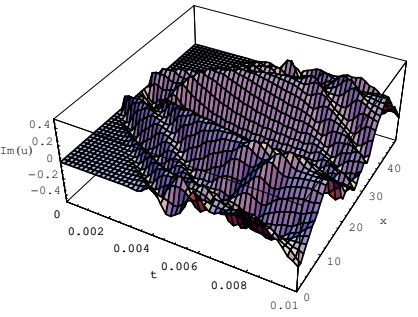

(b) Method $[\mathrm{P}]$.

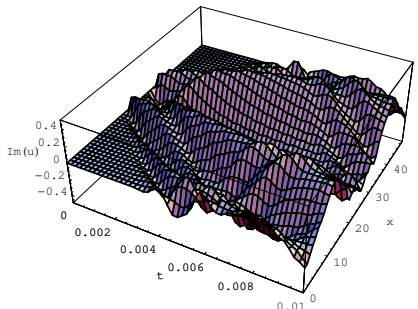

(c) Method [RK].

FIG. 4.3. The imaginary part of the solution for a square pulse initial condition.

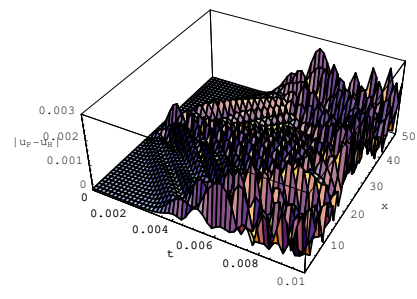

(a) $\left|u_{P}-u_{H}\right|$.

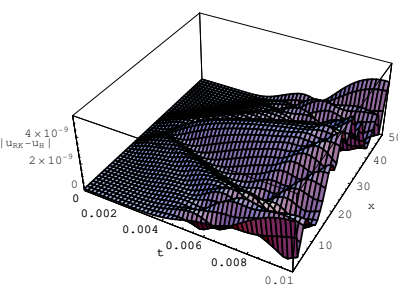

(b) $\left|u_{R K}-u_{H}\right|$.

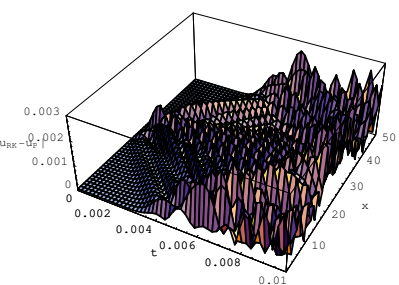

(c) $\left|u_{R K}-u_{P}\right|$.

FIG. 4.4. Differences between the three solutions.

be zero for this recurrent solution. We set $\alpha:=1, N:=50, \Delta t:=1 / 2000$, and $t \in[0,10]$. 


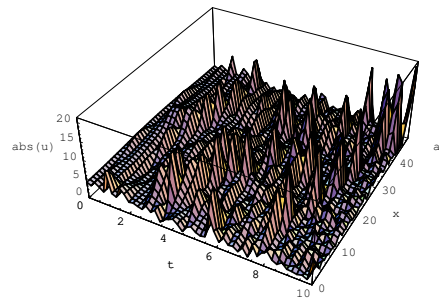

(a) Method $[\mathrm{H}]$.

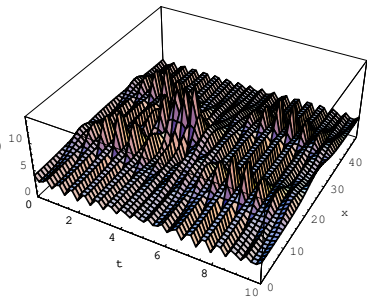

(b) Method [P].

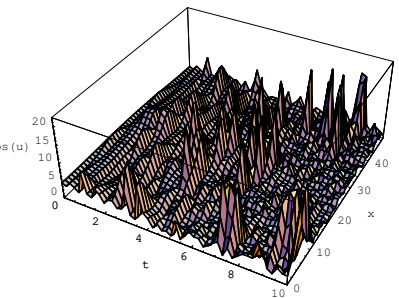

(c) Method $[\mathrm{RK}]$.

FIG. 4.5. The absolute value of the solution for the recurrence $(\Delta t=1 / 2000)$.

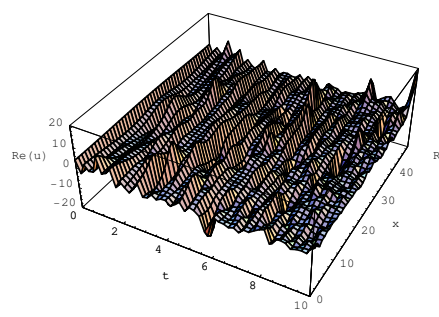

(a) Method $[\mathrm{H}]$.

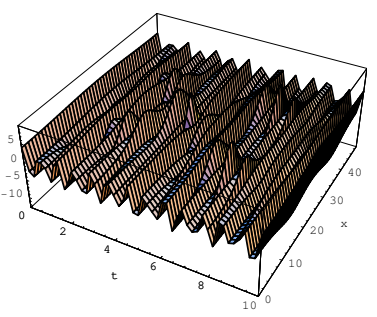

(b) Method [P].

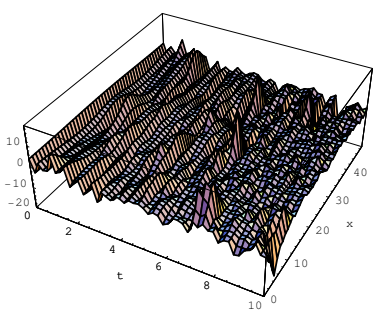

(c) Method $[\mathrm{RK}]$.

FIG. 4.6. The real part of the solution for the recurrence $(\Delta t=1 / 2000)$.

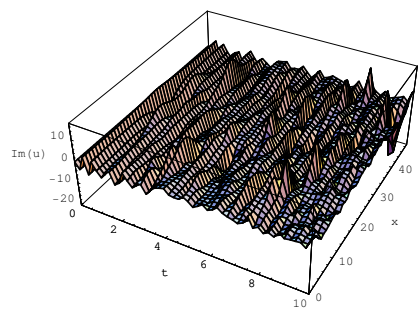

(a) Method $[\mathrm{H}]$.

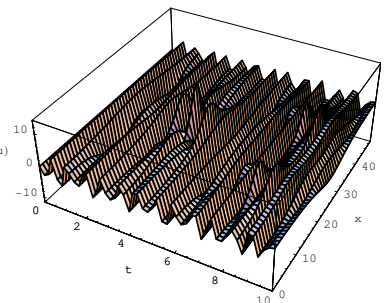

(b) Method [P].

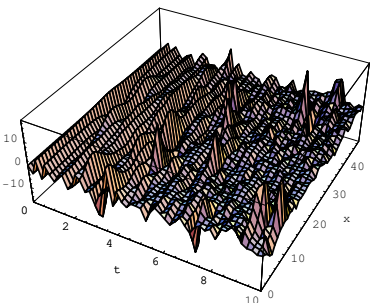

(c) Method $[\mathrm{RK}]$.

FIG. 4.7. The imaginary part of the solution for the recurrence $(\Delta t=1 / 2000)$.

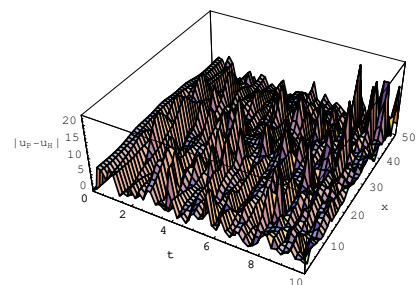

(a) $\left|u_{P}-u_{H}\right|$.

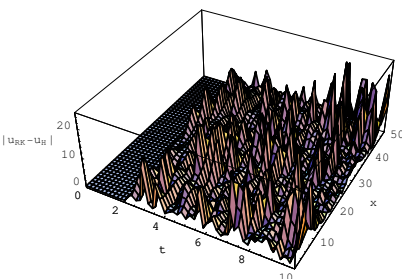

(b) $\left|u_{R K}-u_{H}\right|$.

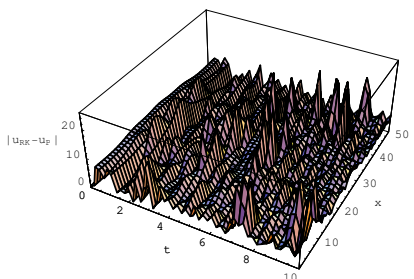

(c) $\left|u_{R K}-u_{P}\right|$.

FIG. 4.8. Differences between the three solutions $(\Delta t=1 / 2000)$.

Figures 4.5, 4.6, and 4.7 show the solutions obtained by the three methods. Method $[\mathrm{P}]$ shows recurrent behavior, whereas methods $[\mathrm{H}]$ and $[\mathrm{RK}]$ show "ran- 


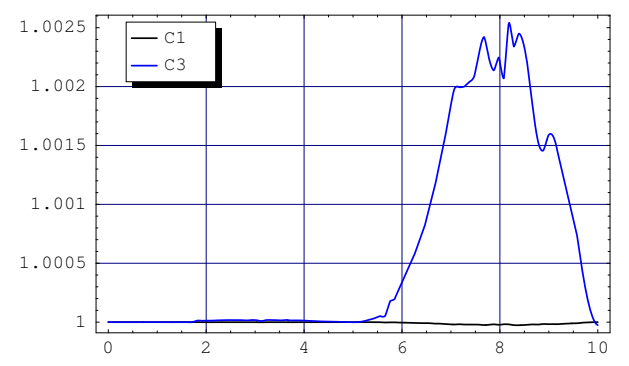

(a) Method [H]: $C_{1}(t) / C_{1}(0)$ and $C_{3}(t) / C_{3}(0)$.

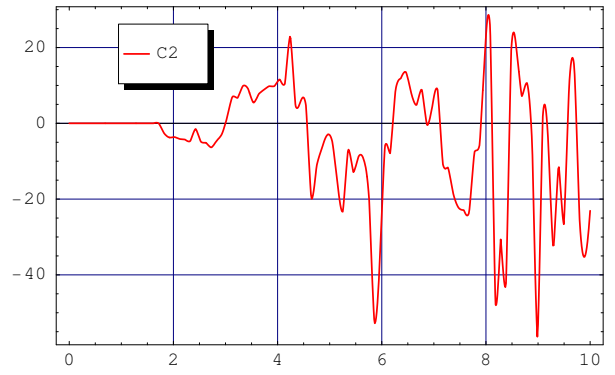

(b) Method $[\mathrm{H}]: C_{2}(t)$.

FIG. 4.9. The time evolution of the conservation laws $C_{1}$ (charge conservation), $C_{2}$ (momentum conservation), and $C_{3}$ (energy conservation) for method $[H](\Delta t=1 / 2000)$. (a) shows the relative changes of $C_{1}(t)$ and $C_{3}(t)$ compared to the initial value, and (b) shows the absolute change in $C_{2}(t)$ which is zero initially.

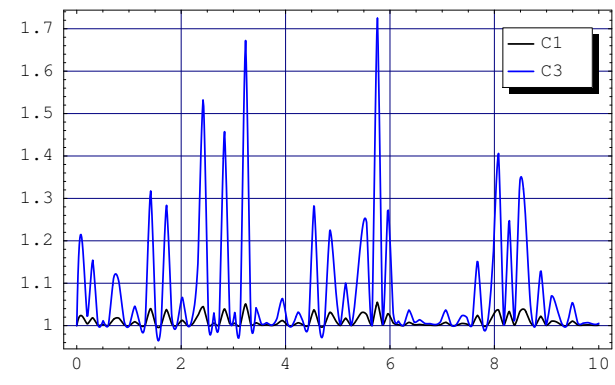

(a) Method $[\mathrm{P}]: C_{1}(t) / C_{1}(0)$ and $C_{3}(t) / C_{3}(0)$.

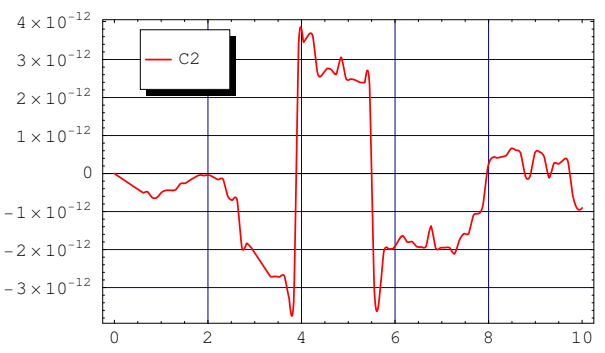

(b) Method $[\mathrm{P}]: C_{2}(t)$.

FIG. 4.10. The time evolution of the three conservation laws for method $[P](\Delta t=1 / 2000)$.

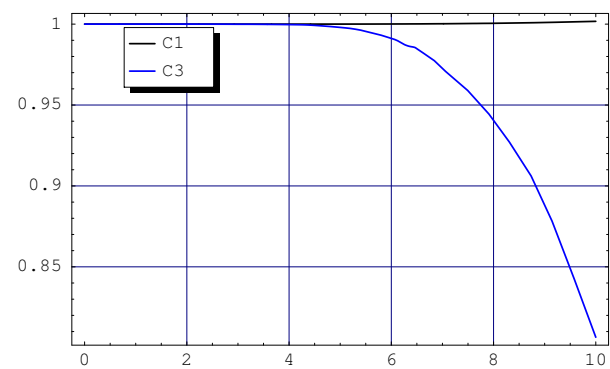

(a) Method [RK]: $C_{1}(t) / C_{1}(0)$ and $C_{3}(t) / C_{3}(0)$.

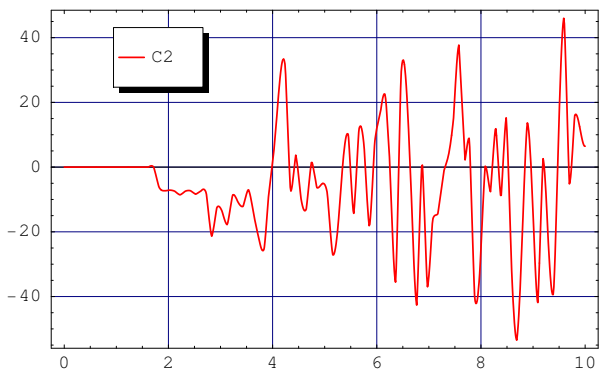

(b) Method [RK]: $C_{2}(t)$.

FIG. 4.11. The time evolution of the three conservation laws for method $[R K](\Delta t=1 / 2000)$.

dom" behavior after $t \approx 6$ and $t \approx 4$, respectively. Fig. 4.8 shows the differences between the three solutions. It is noted that up to $t \approx 2$, methods $[\mathrm{H}]$ and $[\mathrm{RK}]$ agree well. 


\begin{tabular}{lllll} 
& & Method $[\mathrm{H}]$ & Method $[\mathrm{P}]$ & Method $[\mathrm{RK}]$ \\
\hline Charge: & $\Delta C_{1}$ & $\approx 5.18626 \cdot 10^{-4}$ & $\approx 1.10087 \cdot 10^{0}$ & $\approx 3.41766 \cdot 10^{-2}$ \\
Momentum: & $\Delta C_{2}$ & $\approx 8.21209 \cdot 10^{+1}$ & $\approx 6.98945 \cdot 10^{-12}$ & $\approx 9.98299 \cdot 10^{+1}$ \\
Energy: & $\Delta C_{3}$ & $\approx 1.02975 \cdot 10^{0}$ & $\approx 2.93506 \cdot 10^{+2}$ & $\approx 7.74859 \cdot 10^{+1}$
\end{tabular}

TABLE 4.1. Summary of the deviations of the conservation properties for all three methods.

The time evolution of the conservation laws $C_{1}, C_{2}$, and $C_{3}$ on the interval $t \in$ $[0,10]$ for all three solutions is shown in Figures 4.9, 4.10, and 4.11. In the cases of $C_{1}$ and $C_{2}$, the relative changes with respect to the initial value are plotted, and in the case of $C_{3}$ the absolute change is depicted. It is noted that both methods $[\mathrm{H}]$ and [RK] conserve $C_{1}$ well. Starting at $t \approx 5,[\mathrm{H}]$ and [RK] do not conserve $C_{3}$ any more: while the relative deviation of method $[\mathrm{H}]$ is $\approx 0.25 \%$, the deviation of method $[\mathrm{RK}]$ is much larger. Regarding $C_{2}$, methods $[\mathrm{H}]$ and [RK] conserve $C_{2}$ up to $t \approx 2$ and show a large, oscillating deviation afterwards. This behavior is consistent with the difference between the two methods shown in Fig. 4.8(b), where both solutions agree well until $t \approx 2$. Interestingly enough, method $[\mathrm{H}]$, a symplectic method, and method [RK], a non-symplectic one, show qualitatively very similar behavior regarding the conservation laws.

The situation for method $[\mathrm{P}]$ is very different from the other two methods. There are fluctuations in the relative values of $C_{1}$ up to $\approx 5 \%$ where $C_{1}$ is too large and fluctuations in the relative values of $C_{3}$ with many peaks over $10 \%$ and one peak reaching $70 \%$. These fluctuations start immediately. At the same time, the absolute deviation of $C_{2}$ is less than $2 \cdot 10^{-12}$. Thus $C_{2}$ here is the only quantity of all three methods that is preserved to almost round-off accuracy. Considering all three conservation laws, method $[\mathrm{P}]$ is not advantageous compared to the other two methods.

Table 4.1 summarizes the results of the numerical experiments. The columns in this table contain an approximation of

$$
\Delta C_{j}:=\max _{t \in\left[0, t_{\max }\right]} \int C_{j}(x) \mathrm{d} x-\min _{t \in\left[0, t_{\max }\right]} \int C_{j}(x) \mathrm{d} x .
$$

It is seen that each of the three methods obeys one conservation law well (nearly exactly in the case of $C_{2}$ by $[\mathrm{P}]$ ), but fails on the rest of the conservation laws.

\section{Conclusion}

Interpreting the numerical results, we observe the following. Although method $[\mathrm{P}]$ is the most advanced method from a theoretical point of view, its conservation properties are not better than those of the other two methods. Probability and energy are not conserved by $[\mathrm{P}]$ and oscillations start immediately; on the other hand, momentum is conserved. The error in energy conservation reaches more than $70 \%$ at one point (cf. Fig. 4.10). A serious disadvantage of method $[\mathrm{P}]$ is that it cannot be applied to soliton solutions, because this would imply a division by zero (see the derivation of the scheme in Section 3.2).

Methods $[\mathrm{H}]$ and $[\mathrm{RK}]$ yield comparable results. Both methods conserve all three quantities reasonably well up to $t \approx 2$. Interestingly, methods $[\mathrm{H}]$ and $[\mathrm{RK}]$, i.e., a symplectic and a non-symplectic scheme, exhibit qualitatively similar behavior, 
although the drift in energy is more pronounced for $[\mathrm{RK}]$ as time progresses. In our implementation, $[\mathrm{RK}]$ works much faster than $[\mathrm{H}]$, and $[\mathrm{P}]$ is the slowest method.

Tracking the conservation laws is a good way of assessing the numerical quality of a solution. It is found that a FD scheme derived by writing the Schrödinger equation as an (artificial) Hamiltonian system does not necessarily conserve important (physical) quantities better than other methods; in fact, this scheme performs worst in the sense that oscillations start immediately. This is due to the fact that the Hamiltonian form is arbitrary. Although the Hamiltonian is conserved by the symplectic integrators, other physical quantities are not necessarily conserved and the Hamiltonian does not generally carry a physical meaning in contrast to the conservation laws derived by Lie theory.

\section{REFERENCES}

[1] M. Ablowitz and J. Ladik, A nonlinear difference scheme and inverse scattering, Studies in Appl. Math., 55, 213-229, 1976.

[2] N. Akhmediev, Nonlinear physics-déjà vu in optics, Nature, 413, 267-268, 2001.

[3] E. Fermi, J. Pasta and H.C. Ulam, in Collected Papers of Enrico Fermi, E. Segrè, ed., The University of Chicago, Chicago, 2, 977-988, 1965.

[4] B. Fornberg, A Practical Guide to Pseudospectral Methods, Cambridge University Press, Cambridge, 1996.

[5] E. Hairer, C. Lubich and G. Wanner, Geometric Numerical Integration: Structure-Preserving Algorithms for Ordinary Differential Equations, Springer-Verlag, Berlin, 2002.

[6] C. Heitzinger and C. Ringhofer, A note on the symplectic integration of the nonlinear Schrödinger equation, Journal of Computational Electronics, 3, 33-44, 2004.

[7] S. Lie, Gesammelte Abhandlungen, 5. Band: Abhandlungen über die Theorie der Transformationsgruppen, B. Teubner, Leipzig, 1924.

[8] E. Noether, Invariante Variationsprobleme, Nachr. König. Gesell. Wissen. Göttingen, Math.Phys. Kl., 2, 235-257, 1918.

[9] P. Olver, Applications of Lie Groups to Differential Equations, Springer-Verlag, 2nd ed., 1993.

[10] G.V. Simaeys, P. Emplit and M. Haelterman, Experimental demonstration of the Fermi-PastaUlam recurrence in a modulationally unstable optical wave, Phys. Rev. Lett., 87(3), 0339021-033902-4, 2001. 